\title{
OOFORECTOMIA EM CORN SNAKE (Pantherophisguttatus): RELATO DE CASO
}

\author{
Oophorectomy in corn snake (Pantherophisguttatus): case report
}

\section{(D)Alexandre Esteves Vieira ${ }^{1}$ (D) Flávio da Silva Nunes ${ }^{1}$ Mariana Gonçalves de Andrade Paiva ${ }^{1}$}

${ }^{1}$ Centro Universitário de Valença (UNIFAA) Valença (RJ)

\section{Autor correspondente:}

Alexandre Esteves Vieira

E-mail: mvalexandreesteves@gmail.com

\section{Como citar este artigo:}

VIEIRA, A. E.; NUNES, F.S.; PAIVA, M. G. A. Ooforectomia em corn snake (Pantherophisguttatus): relato de caso. Revista Saber Digital, v. 14, n. 2, p. 69-79, 2021.

Data de submissão: 26/05/2021

Data de aprovação: 29/06/2021

Data de publicação: 21/08/2021

\section{(c) (1) (8)}

Esta obra está licenciada com uma licença

http://creativecommons.org/licenses/by-nc/4.0/

\section{RESUMO}

Introdução: Ooforectomia é o procedimento cirúrgico que consiste na retirada dos ovários e ovidutos dos répteis, sendo responsável pela esterilização do animal e possível aliado no controle de natalidade da Pantherophis guttatus, também conhecida como Corn Snake, espécie de serpente exótica, criada como pet, que vem sendo reproduzida e comercializada de maneira indiscriminada em território brasileiro. Objetivo: relatar o protocolo anestésico multimodal e a técnica cirúrgica de Ooforectomia em um exemplar de Corn Snake. Relato de caso: Após constatar estado de higidez, o animal foi contido quimicamente com a associação de Cetamina $1 \mathrm{mg} / \mathrm{kg}$ Midazolam $1 \mathrm{mg} / \mathrm{kg}$ e Cloridrato de Nalbufina $2 \mathrm{mg} / \mathrm{kg}$, aplicados pela via intramuscular. A indução e manutenção anestésica foram feitas com anestésico inalatório Isoflurano, concebendo ao animal o máximo de conforto durante o procedimento cirúrgico. Com o animal em plano anestésico cirúrgico, foi realizado o bloqueio local infiltrativo em plano subcutâneo, na linha de incisão utilizando a Bupivacaína como anestésico local. A técnica cirúrgica de ooforectomia resumese na extração total dos ovários e ovidutos através de uma celiotomia paraventral na altura dos órgãos em questão, incapacitando a reprodução do animal, alcançando o controle de natalidade e evitando possíveis problemas relacionados a ovipostura. Conclusão: A esterilização do espécime através da realização da técnica cirúrgica de ooforectomia, associadas aos cuidados trans e pós operatório são eficazes para a manutenção do estado de saúde do animal e controle populacional.

Palavras-chave: Anestesia, esterilização, desequilíbrios ambientais, espécie exótica, controle de natalidade.

\section{ABSTRACT}

Introduction: Oophorectomy is the surgical procedure that consists of removing the ovaries and oviducts of reptiles, being responsible for the sterilization of the animal and a possible ally in the birth control of Pantherophis guttatus, also known as Corn Snake, a species of exotic snake, bred as an animal of estimation, which has been reproduced and marketed indiscriminately in Brazilian territory. Objective: to report the multimodal anesthetic protocol and the surgical technique of Oophorectomy in a specimen of Corn snake. Case report: After verifying the state of health, the animal was chemically contained with the association of Ketamine $1 \mathrm{mg} / \mathrm{kg}$, Midazolam $1 \mathrm{mg} / \mathrm{kg}$ and Nalbuphine Hydrochloride $2 \mathrm{mg} / \mathrm{kg}$, expressed by intramuscular route. Anesthetic induction and maintenance were performed with an Isoflurane inhalation anesthetic, providing the animal with maximum comfort during the surgical procedure. With the animal in the surgical anesthetic plane, a local infiltrative block was performed in the subcutaneous plane, at the incision line, using a Bupivacaine as a local anesthetic. The surgical technique of oophorectomy resumes the total extraction of the ovaries and oviducts through a paraventral celiotomy at the level of the organs in question, incapacitating the animal's reproduction, achieving birth control and avoiding possible problems related to oviposition. Conclusion: The sterilization of the specimen by performing the surgical technique of oophorectomy, associated with trans and post-operative care are good for the maintenance of the animal's health status and population control.

Keywords: Anesthesia, sterilization, environmental imbalances, exotic species, birth control. 


\section{INTRODUÇÃO}

As serpentes são pertencentes à ordem Squamata e componentes da Subordem Serpentes. Há atualmente aproximadamente 2900 espécies ao redor do globo terrestre, que habitam quase todas as localidades do planeta, principalmente as regiões tropicais (GREGO; ALBUQUERQUE E KOLENIKOVAS, 2014).

As serpentes, como todos os outros répteis são animais ectotérmicos, precisam de fontes externas de calor para elevar e manter a sua temperatura corporal constante, sendo essa uma característica com ligação direta com os aspectos de sua fisiologia, afetando em fatores como comportamento reprodutivo, metabólico e alimentar (MOSLEY, 2005).

Os procedimentos cirúrgicos em répteis estão em constante evolução e novas técnicas cirúrgicas tem sido relatadas nos últimos anos. Esses procedimentos inovadores geralmente reduzem a utilização de intervenções convencionais ou fornecem soluções perspicazes para distúrbios comuns (DI GIROLAMO; MANS,2016).

Nas serpentes, a realização de procedimentos clínicos e cirúrgicos necessitam aplicação de contenção química e anestesia. Para a escolha do protocolo anestésico, deve se levar em consideração fatores como o estado físico do animal, procedimento cirúrgico que será realizado e sua duração (VALENTE; BIANCHI; CONTESINI, 2013).

O tratamento médico cirúrgico subsequente associado a cada afecção é de grande importância em alguns distúrbios reprodutivos, como a estase pré e pós-ovulatória, ovos ectópicos e neoplasias (MANS;SLADKY,2012). Técnicas cirúrgicas que tem como objetivo a esterilização de répteis, além de serem utilizadas para procedimentos destinados à correções de afecções do trato reprodutivo, também podem ser realizadas para controle de natalidade, conservação e para atender necessidades ecológicas (RIVERA et al,2014), como em casos da Pantherophis guttatus, também conhecida como cornsnake, 
serpente proveniente dos Estados Unidos que vem sendo criada cada vez mais como animal de estimação aqui no Brasil, devido ao alto valor comercial de espécies silvestres provenientes de criadouros legalizados, facilidade de manejo e alta variabilidade de padrões de cores (MACEDO,2018).

Fisiologicamente, o trato reprodutivo de répteis fêmeas é composto por dois ovários e dois ovidutos. Os ovários são suspensos por um mesovário, que exibem mudanças sazonais, atingindo um tamanho maior durante a estação de reprodução. Os tamanhos dos folículos ovarianos podem afetar significativamente a anatomia e as técnicas cirúrgicas utilizadas, isso porque as serpentes apresentam uma assimetria exagerada entre os ovários, sendo o direito geralmente maior e mais deslocado anteriormente do que o esquerdo (DI GIROLAMO; MANS,2016).

\section{RELATO DE CASO}

Uma serpente da espécie Corn Snake de 2 anos e 8 meses de idade, pesando $450 \mathrm{~g}$ e com 1,20 metros de comprimento foi submetida a cirurgia eletiva de ooforectomia na Policlínica Veterinária Escola do Centro Universitário de Valença (UNIFAA). O animal passou por uma avaliação pré-anestésica, onde foi realizada a aferição dos parâmetros vitais e ultrassonografia com objetivo de qualificar e quantificar os riscos anestésico-cirúrgicos.

Após a realização da avaliação pré-anestésica e confirmação do estado de higidez, o animal foi contido quimicamente com uma associação de Cetaminana dose de $1 \mathrm{mg} / \mathrm{kg}$, Midazolam na dose de $1 \mathrm{mg} / \mathrm{kg}$ e Cloridrato de Nalbufina na dose de $2 \mathrm{mg} / \mathrm{kg}$, pela via intramuscular (Figura 1-A). Após 15 minutos pode-se observar ausência de reflexo de posicionamento e relaxamento muscular do animal, porém não suficiente para intubação orotraqueal, sendo necessário a indução com Isoflurano em 1 de concentração alveolar mínima (CAM), através de máscara facial, durante 3 minutos. A obtenção do relaxamento mandibular total permitiu a intubação orotraqueal por visualização direta, de forma tranquila e sem intercorrências, com a utilização de uma sonda uretral 
número 6 (Figura 1-B), sendo a manutenção anestésica realizada através de anestesia inalatória com Isoflurano em 0,5 CAM e uma fração inspirada de oxigênio $\left(\mathrm{FIO}_{2}\right)$ de $100 \%$. Em seguida foi realizada a antissepsiada da área cirúrgica e o bloqueio local infiltrativo na linha de incisão, em plano subcutâneo com Bupivacaína 0,25\% na dose de $2 \mathrm{mg} / \mathrm{kg}$. Foram utilizados como parâmetros de monitoração eletrocardiograma (ECG) (Figura 1-C), oximetria de pulso $\left(\mathrm{SPO}_{2}\right)$ (Figura 1-D), capnografia $\left(\mathrm{ETCO}_{2}\right)$ (Figura 1-E) e Doppler vascular (Figura 1-E). Durante todo o procedimento cirúrgico o animal manteve uma média dos parâmetros vitais de $\mathrm{FC}=80 \mathrm{bpm}, \mathrm{FR}=2 \mathrm{rpm}, \mathrm{SPO} 2=98 \%$ e ETCO2 $=47 \mathrm{mmHg}$. $O$ plano anestésico foi monitorado através da progressão do relaxamento muscular, reflexo de reposicionamento e o reflexo de dor por pinçamento.

A técnica cirúrgica começou com uma celiotomia paraventral, sendo a incisão realizada ao nível dos ovários e ovidutos, entre a primeira e a segunda fileira de escamas laterais, evitando a distorção das escamas ventrais quando a pele fosse suturada. Os ovidutos, que estavam repletos de ovos em calcificação, o que ajudou a visualização, foram suavemente exteriorizados, ovários identificados e elevados, expondo o mesovário com seus vasos (Figura 1-F). Os vasos que suprem o oviduto foram cuidadosamente ligados utilizando fio absorvível poligalactina 2.0. A ligadura foi feita distalmente, próximo à cloaca e o oviduto removido.

Foram utilizadas suturas evaginantes no padrão Wolf, revertendo a ondulação formada na pele incisada (Figura 1-G).

Após o término do procedimento cirúrgico, que durou 55 minutos, o fluxo de anéstésico inalatório foi cessado, porém o animal permaneceu intubado em $\mathrm{FIO}_{2}=100 \%$ até recuperar seus movimentos, em sentido crânio-caudal, que se deu após 7 minutos de descontinuidade do Isoflurano. O suporte térmico foi mantido durante o procedimento cirúrgico através de colchão térmico e adicionado luvas de látex contendo soro morno após o término.

No pós-operatório foi administrado Meloxicam na dose de 0,2mg/kg SID como controle analgésico durante cinco dias e Enrofloxacina na dose de $5 \mathrm{mg} / \mathrm{kg}$ 
em três aplicações a cada 48 horas para controle preventivo antimicrobiano. Os pontos foram retirados seis semanas após a cirurgia e após a ecdise.

A avaliação da qualidade analgésica foi feita através da monitorização comportamental do animal no pós-operatório imediato e nas primeiras 48 horas de pós-operatório. Durante as primeiras 48 horas o animal apresentou-se letárgico, e nas horas subsequentes foi retornando as atividades e comportamentos normais, se mostrando bastante ativo.

Figura 1 - Sequência de fotos evidenciando vias de administração, monitorização anestésica e técnica cirúrgica.

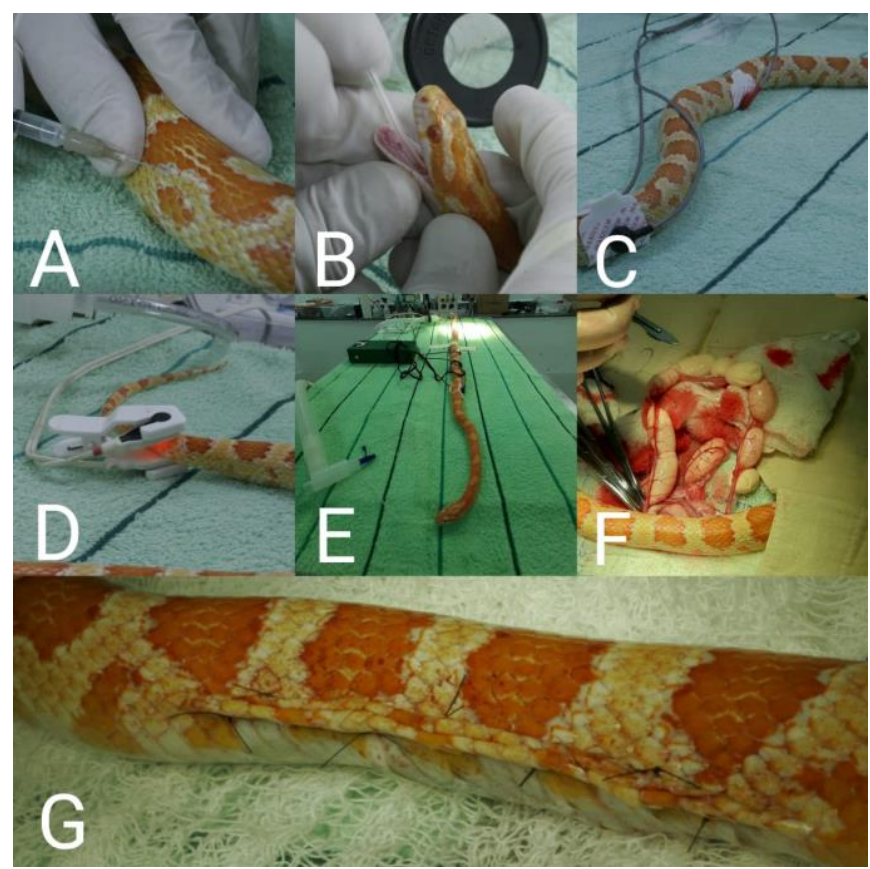

Legenda: A:Contenção química. B:Intubaçãoorotraqueal. C:Monitoração por ECG. D:Monitoração por oximetria. E:Monitoração por Doppler vascular. F:Exteriorização do oviduto.

G: Rafia de pele com pontos em padrão Wolf. Fonte: Arquivo pessoal (2020).

\section{DISCUSSÃO}

A técnica cirúrgica de Ooforectomia é comumente realizada para correção de afecções como estases pré e pós-ovulatória, ovos ectópicos e neoplasias, além de também poder ser utilizada para o controle de natalidade e com 
finalidades de equilíbrio ecológico, de acordo com estudos realizados por Mans e Sladky (2012), e Knafo et al. (2014). Neste relato, o espécime de Corn Snake foi submetido à técnica com o intuito de controle de natalidade, além de tentar conscientizar criadores informais e a população sobre os possíveis desequilíbrios ambientais que podem ser gerados pela má gestão de espécies exóticas como pets.

O estudo da anatomia e fisiologia prévio do grupo e da espécie é essencial, pois são animais extremamente sensíveis aos efeitos adversos dos fármacos anestésicos que, em muitas vezes, não são passíveis de extrapolação alométrica. A utilização da anestesia inalatória com Isofluorano é padrão para serpentes, devido a sua segurança e rápida recuperação. No entanto, essa técnica não abole a utilização de fármacos injetáveis como: anestésicos gerais, analgésicos, sedativos e tranquilizantes que podem ser utilizados como prémedicação, indução e manutenção da anestesia (VALENTE; BIANCHI; CONTESINI, 2013).

Anestésicos locais devem ser utilizados com a finalidade de se propiciar uma analgesia trans e pós-operatória adicional, reduzindo dose dos outros fármacos utilizados e, consequentemente, seus efeitos adversos (VALENTE; BIANCHI; CONTESINI, 2013).Buscamos desta forma a aplicação de uma anestesia multimodal, com o objetivo de potencializar os efeitos dos fármacos e manter o paciente com doses mais seguras.

Segundo DeSimone, Hirano e Santos (2017) assim como nos mamíferos, a administração de benzodiazepínicos em répteis, causa redução da agressividade, relaxamento muscular e alteração psicomotora, porém a administração de 1 e 2 mg/kg de midazolam não foi suficiente para causar uma sedação profunda, sendo necessário para procedimentos invasivos a associação com outros fármacos, como dissociativos e opioides. Os efeitos sedativos dessa associação podem ser avaliados neste relato, sendo considerada eficiente para atingir os resultados esperados.

Os anestésicos dissociativos atuam sobre os receptores N-metil-Daspartato (NMDA), opioides, monoaminérgicos e muscarínicos. A cetamina é um 
agente dissociativo comumente utilizado como parte do protocolo anestésico em répteis, com o objetivo de produzir imobilização e induzir a anestesia. Sua dose efetiva depende da temperatura corporal. As baixas temperaturas corporais requererem baixas doses, mas maiores tempos de indução e de recuperação (ARCOVERDE,2018). Este fármaco foi utilizado na dose de $1 \mathrm{mg} / \mathrm{kg}$, uma dose muito ínfima, pois procuramos buscar mais a sua ação analgésica e sinergismo com os outros associados.

Apesar de difícil percepção, o modo como a dor é demonstrado nas serpentes já foi evidenciado através de componentes anatômicos, fisiológicos e bioquímicos. O não reconhecimento e o desconhecimento de agentes analgésicos, podem resultar em manejo da dor inapropriado. Como o seu reconhecimento ainda é falho nessas espécies, a prevenção é o método mais efetivo para se controlar a dor. Por isso, é preconizado assim como nos mamíferos, protocolos analgésicos balanceados para o controle trans e pósoperatório nas serpentes. Frequentemente, é incluído nestes protocolos a administração de analgésicos sistêmicos como os opioides agonistasantagonistas, como o Butorfanol, sendo o representante mais utilizado dessa classe farmacológica (VALENTE; BIANCHI; CONTESINI, 2013).

No entanto, no que se diz respeito ao Cloridrato de Nalbufina, opioide pertencente à classe dos agonista-antagonista, igualmente ao Butorfanol, não existem estudos clínicos nem farmacodinâmicos que comprovem sua eficácia na espécie em questão.

Completando o protocolo anestésico multimoldal, o bloqueio local realizado com a Bupivacaína, teve como objetivo propiciar uma analgesia de longa duração, visto que seu período hábil é maior do que o produzido pela lidocaína (KLAUMANN; OTERO,2013).

Segundo DiGirolamo e Mans (2016), o procedimento cirúrgico de celiotomia paraventral é indicado para maioria das cirurgias abdominais em serpentes. A incisão precisa ser realizada ao nível do órgão de interesse, a fim de se evitar incisões com extensões desnecessárias. A pele é incisada entre a primeira e a segunda fileira de escamas laterais, o que se permite evitar a 
distorção das escamas ventrais quando a pele for suturada. Neste caso, a incisão foi iniciada à uma distância de $25 \mathrm{~cm}$ da cloaca, onde conseguimos visualizar o oviduto repleto de ovos, expandindo a incisão $5 \mathrm{~cm}$ cranial e $5 \mathrm{~cm}$ caudalmente, ficando uma incisão total de $10 \mathrm{~cm}$ de comprimento.

A técnica de esterilização em serpentes pode mudar de acordo com o ciclo ovulatório. Se a ovulação já ocorreu, os ovidutos são suavemente exteriorizados antes dos ovários. Caso contrário, os ovários são grandes e vários folículos amarelados a alaranjados estão presentes, e sendo estes exteriorizados primeiro que o oviduto. Uma vez que os ovidutos tenham sido exteriorizados, os ovários devem ser identificados e elevados para expor o mesovário com seus vasos. Os vasos que suprem o oviduto são cuidadosamente ligados usando radiocirurgia ou ligaduras. Essa ligadura é feita distalmente, próximo à cloaca e o oviduto removido (DI GIROLAMO; MANS, 2016). No presente caso, a serpente já tinha ovulado e estava com dezesseis ovos em fase de maturação localizados nos ovidutos, então eles foram suavemente exteriorizados antes dos ovários, e posteiormente foram seguidos os passos citados.

Estudos realizados por Garcia e Contesini (2012) mostraram que a sutura de aposição é prática e causa uma cicatriza mais estética, enquanto o padrão de eversão pareceu ingurgitar a pele e desorganizou o padrão linear da distribuição das escamas, sendo os dois padrões de sutura eficazes em serpentes. O padrão de sutura simples se mostrou melhor em quesitos de presença de exsudato, crostas e aderência de ecdises, enquanto o padrão de eversão foi mais eficaz em relação a prevenir infecções secundárias e deiscência de suturas. O padrão de sutura escolhido para o caso foi o de eversão em Wolf, mesmo sabendo dos benefícios estéticos do padrão aposicional, foi escolhido o de eversão, preconizando a prevenção antimicrobiana e evitando a deiscência de sutura.

Para o tratamento pós-operatório, os antiinflamatórios não esteroidais Meloxicam na dose de 0,1 a 0,3mg/kg, por via intramuscular, a cada 24 horas, o carprofeno 1 a $4 \mathrm{mg} / \mathrm{kg}$, por via intramuscular, a cada 24 a 72 horas ou 0 cetoprofeno 1 a $3 \mathrm{mg} / \mathrm{kg}$, por via intramuscular, a cada 24 a 48 horas apresentam 
um efeito satisfatório (VALENTE; BIANCHI; CONTESINI, 2013) e para o controle antimicrobiano, a enrofloxacina é um antibiótico de amplo espectro recomendado contra infecções bacterianas por Acinetobacter, Citrobacterfreundii e Enterobacter em répteis (FERREIRA, et al.2014) na dose de 5 a 10mg/kg por via intramuscular ou oral a cada 48 horas (GREGO; ALBUQUERQUE E KOLENIKOVAS, 2014), corroborando com o protocolo escolhido para manejo pós operatório do animal, que teve uma avaliação analgésica eficiente, visto que o animal supriu suas necessidades fisiológicas de forma independente, tais como ingestão de água e alimento, ecdise e excreção de dejetos, além de se mostrar com comportamento ativo dentro do terrário após 48h corridas do procedimento cirúrgico.

\section{CONCLUSÃO}

No presente relato pode-se concluir que a realização do protocolo anestésico e técnica cirúrgica de Ooforectomia somados aos cuidados antiinflamatórios e antimicrobianos no pós-operatório conferiu ao animal inconsciência, proteção neurovegetativa, miorelaxamento, analgesia trans e pósoperatória e atingiu o objetivo do projeto, que era promover a esterilização do espécime com o intuito de controle de espécies exóticas, evitando possíveis desequilíbrios ambientais.

\section{REFERÊNCIAS}

ARCOVERDE,K.N. Anestesia em répteis com distocia: relato de dois casos. 2018. 38f. Trabalho de Conclusão de Curso (Bacharelado em Medicina Veterinária) - Universidade Federal da Paraíba, João Pessoa, 2018. Disponível em: https://repositorio.ufpb.br/jspui/handle/123456789/4499. Acesso em: 02 nov. 2020.

DE SIMONE, S. B. S; HIRANO, L. Q. L; SANTOS, A. L. Q. EFEITOS DA ADMINSTRAÇÃO DO MIDAZOLAM EM DOSES DIFERENTES EM JIBOIAS Boa constrictor LINNAEUS, 1758 (SQUAMATA: BOIDAE). Ciência Animal 
Brasileira, v. 18, p. 1-10, $2017 . \quad$ Disponível em: https://www.revistas.ufg.br/vet/article/view/e-22230. Acesso em: 02 nov. 2020.

DI GIROLAMO, N.; MANS, C. Reptile Soft TissueSurgery. Veterinary Clinics of NorthAmerica - Exotic Animal Practice, v. 19, n. 1, p. 97-131, 2016.

FERREIRA, P. R. B., et al. Sinais clínicos e alterações necroscópicas em filhotes de Eunectesmurinus (Linnaeus, 1758) infectados com bactérias Gram negativas multirresistentes. JBCA - Jornal Brasileiro de Ciência Animal, v. 7, n. 14, p. 508-522, 2014.

GARCIA, P. B; CONTESINI, E. A. Comparativo entre dois padrões de síntese cutânea e três tipos de materiais de síntese em serpentes Bothropoides jararaca.72 f. 2012. Dissertação (Mestrado em Ciências Veterinárias) Faculdade de Veterinária, Universidade Federal do Rio Grande do Sul, Porto Alegre, 2012 Disponível em: https://www.lume.ufrgs.br/handle/10183/49947. Acesso em: 28 out. 2020.

GREGO, K. F; ALBUQUERQUE, L. R; KOLESNIKOVAS, C. K. M. Ordem Squamata - Subordem Ophidia (Serpente). In: CUBAS, Z. S.; SILVA, J. C. R.; CATÃO-DIAS, J. L. Tratado de animais selvagens. 2.ed. São Paulo: Roca, 2014. p.224-255.

KLAUMANN, P. L.; FILHO, J. C. K; NAGASHIMA, J. K. Anestésicos Locais. In: KLAUMANN, P. L.; OTERO, P. E. Anestesia locorregional em pequenos animais. 1.ed. São Paulo: Roca, 2013. p 23-41.

KNAFO, S. E., et al. Sterilisation of hybrid Galapagos tortoises (Geochelone nigra) for island restoration. Part 1: endoscopic oophorectomy of females under ketamine-medetomidine anaesthesia. Vet Rec, v.168, n.2, p.47, 2011.

MACEDO, D. S. Etno-herpetologia no Recôncavo Baiano: perspectivas e consequências da criação de répteis. 41f. 2018. Trabalho de Conclusão de Curso (Bacharela em Biologia) - Centro de Ciências Agrárias, Ambientais e Biológicas, Universidade Federal do Recôncavo da Bahia, Cruz das Almas, 2018. Disponível em: http://www.repositoriodigital.ufrb.edu.br/bitstream/123456789/1418/1/TCC\%20C orrigido.pdf. Acesso em: 28 out. 2020.

MANS, C., SLADKY, K. K. Diagnosis and management of oviductal disease in three red-eared slider turtles (Trachemys scripta elegans). J Small AnimPract, v.53, n.4, p.234-239, 2012.

MOSLEY, C. A. E. Anesthesia and Analgesia in Reptiles. Seminars in Avian and Exotic Pet Medicine, v.14, n.4, p.243-262, 2005. 
RIVERA, S, et al. Sterilisation of hybrid Galapagos tortoises (Geochelone nigra) for island restoration. Part 2: phallectomy of males under intrathecal anaesthesia with lidocaine. Vet Rec, v.168, n.3, p.168-178, 2011.

VALENTE,F.S., et al. Particularidades na contenção química e na anestesia de serpentes. Veterinária em foco, v.10, n.2, p.210-221, 2013. 